\title{
Desafios e perspectivas do exame preventivo de colo uterino durante a vivência da prática profissional: relato de experiência
}

\author{
Challenges and perspectives of preventive examination of the cervix during the experience \\ of professional practice: experience report
}
Desafíos y perspectivas del examen preventivo del cervix durante la experiencia de la práctica profesional: informe de experiencia

Luan Naís de Souza1*, Tuanny Caroline Pereira de Santana ${ }^{1}$, Anna Luiza Carneiro da Silva ${ }^{1}$, Raila Gonçalves dos Santos ${ }^{1}$, Camilla Talita Silva Canhoto ${ }^{1}$, Jennifer Carla Arruda de Souza ${ }^{1}$, Camilla Sousa Justino da Silva ${ }^{1}$, Joanna Francyne Silva de Barros ${ }^{1}$, Sandra Hipólito Cavalcanti ${ }^{1}$, Maria Celina Matias Rocha'.

\section{RESUMO}

Objetivo: Descrever as experiências durante a prática profissional na realização do exame preventivo de colo uterino, compreendendo seus desafios e perspectivas na saúde pública. Relato de experiência: Trata-se de um estudo descritivo, do tipo relato de experiência, sobre os desafios e perspectivas para o sucesso na realização do exame preventivo de colo uterino, tendo em vista a importância da abordagem adequada e as condutas necessárias para sua execução. $O$ relato ocorreu durante o estágio da Vivência da Prática Profissional (VPP) em saúde da mulher, realizado no período de 04 de março a 26 de abril de 2019. O cenário de estudo foi na citologia oncótica do ambulatório de um hospital escola de referência na cidade do Recife, estado de Pernambuco. O processo de construção do relato teve início após a finalização da VPP, onde deuse início a revisão bibliográfica sobre os desafios da realização do exame do colo do útero e um referencial teórico para essa prática. Considerações finais: Durante o processo de realização dos exames preventivos de colo uterino, houve uma integração de conhecimentos teórico-práticos com validação na formação acadêmica, oportunidade de enfrentar positivamente os desafios e perspectivas da saúde pública que possam Ihe acometer no exercício da enfermagem.

Palavras-chave: Exame papanicolau, Educação em enfermagem, Saúde pública.

\begin{abstract}
Objective: To describe the experiences during professional practice in carrying out the cervical preventive exam, understanding its challenges and perspectives in public health. Experience report: This is a descriptive study, of the type of experience report, about the challenges and perspectives for success in carrying out the cervical preventive exam, in view of the importance of the approach and as necessary conducts for its execution. The report occurred during the stage of the Professional Practice Experience (PPE) in women's health, carried out from March 4 to April 26, 2019. The study scenario was in the oncotic cytology of the outpatient clinic of a reference teaching hospital in Recife city, state of Pernambuco. The process of construction of the report began after the completion of the PPE and maked a bibliographic review of the challenges of performing the cervical exam and a theoretical framework for this practice began. Final considerations: During the process of conducting preventive cervical exams, there was an integration of theoretical and practical knowledge with validation in academic training, an opportunity to positively face the challenges and perspectives of public health that the companion in the exercise of nursing.
\end{abstract}

Keywords: Exame papanicolau, Nursing education, Public health.

${ }^{1}$ Faculdade Pernambucana de Saúde (FPS), Recife - PE. *E-mail: luan4025@hotmail.com 


\section{RESUMEN}

Objetivo: Describir las experiencias durante la práctica profesional en la realización del examen preventivo cervical, entendiendo sus desafíos y perspectivas en salud pública. Informe de experiencia: Se trata de un estudio descriptivo, tipo informe de experiencia, sobre los desafíos y perspectivas para el éxito del examen preventivo de tinción uterina, tomando en cuenta la importancia del abordaje adecuado y las conductas necesarias para su ejecución. El reporte de ocurrencias durante el período de Vida Práctica Profesional (VPP) en salud de la mujer, realizado del 4 de marzo al 26 de abril de 2019. El período de estudios para citología oncótica de un hospital docente ambulatorio de referencia de la ciudad. Recife, estado de Pernambuco. El proceso de construcción de la historia se inició desde el final del VPP, donde se inició la revisión bibliográfica sobre los desafíos de realizar la exploración del útero y un marco teórico para esta práctica. Consideraciones finales: Durante el proceso de realización de dos exámenes preventivos de tinción uterina, se integra los conocimientos teóricos y prácticos con la validación de la formación académica, una oportunidad para afrontar positivamente los retos y perspectivas de salud pública que se pueden realizar sin el ejercicio de enfermedad.

Palabras-clave: Citología vaginal, Educación en enfermería, Salud pública.

\section{INTRODUÇÃO}

O ensino em enfermagem tem sido foco de muitos estudos e debates, principalmente após a instituição das Diretrizes Curriculares Nacionais (DCNs) no ano de 2001, gerando uma série de reestruturações na formação profissional como incorporação do Estágio Curricular Supervisionado (ECS) (ESTEVES LSF, et al., 2018).

No decorrer dos anos, houve o avanço da prática profissional, além do reconhecimento e ética dos procedimentos executados pela enfermagem, de forma integral e holística, como também no papel social e político com a finalidade de reconhecer as individualidades determinantes da saúde pública (FERNANDES JD e REBOUCAS LC, 2013).

O estágio curricular supervisionado durante a graduação em enfermagem é obrigatório. Através dele, os estudantes têm a possiblidade de aplicar o conhecimento teórico durante as atividades práticas, desenvolver de habilidades e atitudes profissionais, e ainda promove reflexão acerca da ação profissional como o fortalecimento de suas competências inseridas nos processos de trabalho das instituições de saúde (NEGREIROS RV, et al., 2018).

O estágio nos campos práticos representa um momento pedagógico importantíssimo para que os estudantes aprendam a problematizar as experiências vividas e desenvolva a capacidade de enfrentar positivamente os desafios da profissão (ESTEVES LSF, et al., 2018). Logo, dentro dos cenários de prática existem áreas de concentração dentre elas a saúde da mulher, que tem como foco proporcionar à mulher uma saúde integral, onde a citologia oncótica vem sendo usada como uma estratégia de ação preventiva de baixo custo e alta eficácia (SILVA ECA, et al., 2016; BRASIL, 2019).

O exame citológico, também conhecido atualmente como Papanicolau é uma análise para investigar alterações nas células do colo uterino, em outras literaturas, este exame pode ser chamado de colpocitologia oncótica cervical e esfregaço cervicorvaginal. Dar-se o nome "Papanicolau" em homenagem ao patologista grego Georges Papanicolau, que estabeleceu em 1942 os conceitos básicos de interpretação citológica e criou um método de coloração citológica que é utilizado, universalmente, até hoje (BRASIL, 2015).

No traçar da revisão da literatura, observou-se que é creditado a Sir Julius Vogel em 1843 o rimeiro relato quanto à aplicação da citologia como meio diagnóstico. Já em 1845, Henri Lebert registrou aspectos morfológico de células aspiradas de tumores. O professor lonel S. Beagle e o Dr. Lambl de Praga em 1850, descreveram células malignas em escarros e urinas. Logo depois em 1853, Donaldson descreveu as características citopatológicas de amostras obtidas da superfície de cortes de tumores (BRASIL, 2012).

Rotineiramente, o exame citopatológico é realizado em serviços de saúde de atenção primária da rede pública por profissionais qualificados. O Papanicolau é responsável pelo rastreamento precoce de lesões 
determinante para o diagnóstico de câncer de colo de útero antes que a mulher apresente os sintomas, porém é necessário que as mulheres recebam informações adequadas sobre a periodicidade desse exame permitindo o diagnóstico precoce (BRASIL, 2015).

O câncer de colo do útero está associado, principalmente, à infecção pelo Papilomavírus Humano (HPV), sendo os mais frequentes os subtipos 16 e 18, representando um grande problema de saúde pública. Essa neoplasia vem sendo apontada como uma das mais importantes preocupações em nível mundial, mesmo apresentando grande potencial de prevenção e cura, quando diagnosticado precocemente (INCA, 2019; NGOMA M e AUTIER P, 2019).

Fatores relacionados ao câncer do colo de útero são cada dia mais estudado. Pesquisas mostram que a imunidade, a genética e o comportamento sexual parecem influenciar os mecanismos ainda incertos que determinam a regressão ou persistência da infecção e também a progressão para lesões precursoras ou o próprio câncer. Em seguida, o tabagismo, precoce, multiplicidade de parceiros sexuais, multiparidade, e, também, o uso de contraceptivos orais são considerados os fatores de maior risco para o desenvolvimento do câncer do colo do útero (BRUNI L, et al., 2019).

O controle e rastreamento do Câncer do Colo do Útero (CCU) é realizado pelo Ministério da Saúde (MS) em conjunto com o Sistema Único de Saúde (SUS) e o Instituto Nacional do Câncer (INCA) (INCA, 2019). Onde, os mesmos orientam para que o rastreamento seja realizado a partir dos 25 aos 59 anos e que contemplem recomendações para sua prevenção, como: rastreamento com o teste de Papanicolau, periodicamente a cada três anos e anualmente de mulheres portadoras do HIV ou imunodeprimidas (INCA, 2016; BRASIL, 2019).

O monitoramento das mulheres ocorre através do Sistema de Informação do câncer do colo do útero (SISCOLO versão 4.18) esse sistema é desenvolvido pelo Departamento de Informática do Sistema Único de Saúde (DATASUS) em parceria com o INCA, para auxiliar a estruturação do Viva Mulher (Programa Nacional de Controle do Câncer do Colo do Útero e de Mama). Coleta e processa informações sobre a identificação de pacientes e laudos de exames citopatológicos e histopatológicos, fornecendo dados para o monitoramento externo da qualidade dos exames, e assim orientando os gerentes estaduais do Programa sobre a qualidade dos laboratórios responsáveis pela leitura dos exames no município (SISCOLO, 2012).

Desta maneira, o objetivo desse estudo é descrever as experiências durante a vigência da prática profissional na realização do exame preventivo do colo uterino, compreendendo seus desafios e perspectivas na saúde pública.

\section{RELATO DE EXPERIÊNCIA}

Trata-se de um estudo descritivo do tipo relato de experiência vivenciado por estudantes do $7^{\circ}$ semestre do Curso da Graduação em Enfermagem de uma Instituição de Ensino Superior (IES) do Recife, durante as atividades curriculares correspondentes ao eixo Introdução à Enfermagem em Saúde da Mulher, ocorrido de março a abril de 2019.

O relato foi desenvolvido no ambulatório de um hospital escola de referência na cidade do Recife, estado de Pernambuco. O público foi composto de mulheres acompanhadas e que realizam consultas mensalmente. Isto posto, são disponibilizadas 80 fichas diárias, sendo 40 no turno da manhã e 40 pelo turno da tarde, onde o atendimento será por onde de chegada de acordo com a numeração das fichas marcadas. O processo de construção do relato teve início após a finalização da Vivência da Prática Profissional (VPP) da IES no hospital escola, das quais, os estudantes realizam as atividades práticas. No andamento do (VPP) deu-se início a revisão bibliográfica sobre os desafios da realização do exame do colo do útero durante a graduação e buscando-se um referencial teórico que fosse capaz de embasar o conhecimento técnico-científico quando pelo olhar humanizado e holístico dos estudantes diante desta prática tão comum na enfermagem.

Mediante a revisão profunda sobre a construção e as técnicas envolvidas para realização do exame de papanicolau, os estudantes aplicaram em sua prática o exame físico e a anamnese, técnicas propedêuticas essenciais para o desenvolvimento desse procedimento. O desenvolvimento dessa prática, foi essencial para 
que os estudantes conseguissem contemplar o Conhecimento, Habilidade e Atitude (CHA) (NEGREIROS RV, et al., 2018).

As atividades foram realizadas num período de cinco semanas, na qual, a primeira etapa consistiu na observação da realidade e identificação de possíveis problemas mediante a participação ativa dos estudantes no acolhimento. $\mathrm{O}$ acolhimento contou com dois momentos: extra consulta e intra consulta.

$\mathrm{Na}$ extra consulta trabalhou-se a educação em saúde por meio de palestras dialogadas que corroborou para esclarecer dúvidas e aproximação com o público. Em seguida, na intra consulta, ressaltou-se a peculiaridade de cada paciente durante a requisição do exame histopatológico de colo uterino. Sendo assim, possível torná-la parte integrante durante o exame.

\section{DISCUSSÃO}

Os estudantes realizam as atividades práticas através da supervisão e orientação da enfermeira da citologia do hospital escola, onde essa preceptora induz os estudantes a desenvolverem o senso crítico reflexivo durante o estágio. Visando uma maior expansão dos conhecimentos, é realizado um feedback dos exames feitos durante as consultas, conforme cada caso e, também, interpretações de resultados de exames anteriores.

A partir dos momentos vivenciados durante o estágio, foram adquiridas diversas experiências, houve 0 aprimoramento do pensamento crítico-reflexivo e senso de responsabilidade, desenvolvimento de habilidades e atitudes no que se refere ao exame Papanicolau, entendendo os desafios que o cenário real traz para a prática profissional. Além de expandir nossos olhares em relação ao setor de citologia e as diversas situações que podem ser encontradas nesse ambiente hospitalar, enquanto estudante de enfermagem, tornou-se possível a capacidade de lidarmos com situações-problemas a qual permite um crescimento acadêmico, uma maneira simplificada de compartilhar conhecimento com os profissionais, estudantes e clientes (BRASIL, 2016).

O estudo da citologia vem possibilitando muitos benefícios para as mulheres que realizam o exame precocemente. Através dele é possível examinar células coletadas do colo do uterino, que tem por finalidade a detecção do câncer de colo de útero em estágio inicial ou anormalidades nas células que podem estar associadas ao desenvolvimento deste tipo de tumor como: infecções no colo de útero ou na vagina como herpes, verrugas genitais, Vírus do Papiloma Humano (HPV) (SILVA ECA, et al., 2016).

Na IES, existe a inserção precoce ao cenário de Prática na Atenção Primária (PAP), onde é permitido ao estudante a observação e/ou participação emprocedimentos mais simples, porém de extrema relevância para a saúde da mulher, que nos possibilitam iniciar o senso crítico e reflexivo para resolução de situações problemas. Consequentemente, o hospital-escola, por ser um de referência e oferecer serviços ambulatoriais, os estudantes já conseguem desempenhar tais atividades mais facilmente.

O exame de papanicolau está intrinsicamente ligado ao bem-estar e saúde da mulher, de forma que os benefícios a curto e longo prazo são reconhecidos pelas principais organizações de saúde. Logo, se faz necessário o conhecimento teórico-prático referente aos exames citológicos que é indispensável para 0 profissional da área de saúde, o qual irá ocupar-se com a saúde da mulher, seja na atenção primária ou em serviços hospitalares que desenvolvem assistência a esse grupo.

Existe uma série de fatores que envolvem o exame, como as principais indicações para fazê-lo, o intervalo ideal entre as coletas, como a mulher deve se preparar para realizá-lo, o que ocorre durante a realização do procedimento, os materiais necessários, o que se espera após a realização do exame e outros. Tudo isso requer do enfermeiro um domínio sobre o conhecimento das estruturas anatômicas da genitália feminina, sabendo distinguir os achados normais dos anormais, durante a coleta das amostras.

Isto posto, encontram-se partes anatômicas importantíssimas e que devem ser avaliadas durante esse exame do colo de útero como: o útero, a vagina e o conjunto dos órgãos genitais externos: monte do púbis, lábios maiores, lábios menores, hímen, prepúcio do clitóris, clitóris, páreo externo da uretra e óstio externo da vagina (PAULSEN F e WASCHKE J, 2018). Tal qual, se faz necessário a inserção de estudantes durante a 
graduação em cenários de práticas que abordem essa temática, bem como, preceptores capacitados para guiar o aprendizado dos acadêmicos neste momento.

O estágio é uma forma eficaz de oferecer contato direto com a realidade, estudantes e profissionais vinculados acreditam que o exame deve ser levado a sério, mas acima de tudo, conduzido por profissionais preparados para executá-lo de forma correta, além de oferecer um acolhimento apropriado, que resulte numa coleta adequada, proporcionando, por fim, a análise laboratorial desejada.

A supervisão durante a realização do estágio visa contribuir de forma integral para qualidade desse futuro profissional que irá ser formado, trabalhando suas inseguranças e suas concepções. Proporcionando qualidade da assistência prestada ao cliente, redução de erros durante procedimentos, prática honesta e contribuindo para satisfação do cliente e família. Também, a instituição de ensino desempenha um papel social de ensino e corresponsável pela formação do discente (COFEN, 2018; OLIVEIRA RS, et al., 2018).

É importante que haja uma adesão significativa para o desenvolvimento de habilidades e atitudes durante a vivência da prática profissional e, para isso, é necessária uma parceria entre o ensino e os serviços de saúde para superar os desafios nessa formação, sendo evidente a necessidade de investimentos em espaços educativos, além de novos recursos e programas inovadores (TEIXEIRA E, 2017).

Dentre as habilidades e competências imprescindíveis a formação do estudante estão, autonomia, liberdade, criatividade, responsabilidade, ética, compromisso, domínio da prática e seu papel social, além do aprofundamento e contextualização dos conhecimentos adquiridos durante a sua formação ligado a forma de atuar do enfermeiro preceptor (TAVARES PN, et al., 2011).

A inserção precoce do estudante de enfermagem proporciona uma formação holística, reflexiva e crítica para o desenvolver de um cuidado humanizado, diferenciando-se do ensino tradicional fragmentado e especializado, onde o contato com a clientela acontecia numa etapa avançada da formação (MARTINS MG, et al., 2001).

O estágio no decorrer da graduação valida o curso, propiciando ao estudante a oportunidade de enfrentar positivamente os desafios da futura profissão, estabelecendo artifícios para que lide com as inúmeras situações que possam lhe acometer no exercício da enfermagem. Durante o estágio no setor da citologia oncótica cervical no ambulatório do hospital-escola na cidade do Recife, estado de Pernambuco, houve uma integração de conhecimentos teórico-prático tratando-se do exame Papanicolau e suas implicações, além do aperfeiçoamento das habilidades práticas para realizar a correta coleta do exame, houve também a educação em saúde para as mulheres sobre a prevenção do câncer do colo do útero e infecções sexualmente transmissíveis. A experiência teórico-prática vivenciada no estágio proporcionou novas formas de pensar e agir diante de práticas relacionadas à saúde da mulher.

\section{REFERÊNCIAS}

1. BRASIL. Ministério da Saúde. Protocolos da Atenção Básica: Saúde das Mulheres / Ministério da Saúde, Instituto Sírio-Libanês de Ensino e Pesquisa - Brasília: Ministério da Saúde, 2016.

2. BRASIL. Ministério da Saúde. Secretaria de Atenção à Saúde. Departamento de Atenção Básica. Rastreamento (Série A: Normas e Manuais Técnicos. Cadernos de Atenção Primária n29). Brasília, 2010.

3. BRASIL. Ministério da Saúde. Citologia em meio líquido para rastreamento de câncer de colo de útero e lesões precursoras- Brasília: Ministério da Saúde, 2019.

4. BRASIL. Ministério da saúde. Papanicolau (exame preventivo de colo de útero). Brasília, 2015.

5. BRASIL. Ministério da saúde. Técnico em citopatologia. Caderno de referência 1: Citopatologia Ginecológica. Brasília, 2012.

6. BRUNI L, et al. ICO/IARC Information Centre on HPV andCancer (HPV Information Centre). Human Papilloma virus and Related Diseases in the World. Summary Report 17 June, 2019.

7. COFEN. Conselho Federal de Enfermagem. PARECER № 02/2018/COFEN/CTLN. Organização de enfermagem. Definição da supervisão de enfermagem, 2018.

8. ESTEVES LSF, et al. Estágio supervisionado na graduação em enfermagem: revisão integrativa. Rev. Bras. Enferm., Brasília, 2018; 71(4): 1740-1750.

9. FERNANDES JD, REBOUCAS LC. Uma década de Diretrizes Curriculares Nacionais para a Graduação em Enfermagem: avanços e desafios. Rev. bras. enferm., Brasília, 2013; 66: 95-101. 
10. FREBRASGO. Federação Brasileira das Associações de Ginecologia e Obstetrícia. Rastreio, diagnóstico e tratamento do câncer de colo de útero. São Paulo, 2017.

11. INCA. Instituto Nacional do Cancêr. Políticas e ações para prevenção do câncer no Brasil. Coordenação de Prevenção e Vigilância. Rio de Janeiro: INCA, 2016.

12. INCA. Instituto Nacional do Cancêr. Parâmetros técnicos para o rastreamento do câncer do colo do útero. Rio de Janeiro: INCA, 2019.

13. MARTINS MG, et al. Avaliação da observação precoce de estudantes universitários em serviços de saúde. R. Bras. Enferm., Brasília, 2001; 54(4): 651-657.

14. MORÁN J. Mudando a educação com metodologias ativas. Coleção Mídias Contemporâneas. Convergências Midiáticas, Educação e Cidadania: aproximações jovens. Vol. II. PG: Foca Foto-PROEX/UEPG, 2015.

15. NEGREIROS RV, et al. Importância do Estágio Supervisionado Para o Acadêmico de Enfermagem no Hospital: Compartilhando Experiência Vivenciadas com a Equipe de Trabalho, 2018; 16(2).

16. NGOMA M, AUTIER P. Cancer prevention: cervical cancer. Ecancermedicalscience. 2019; $13: 952$.

17. OLIVEIRA RS, et al. Atuação do enfermeiro nas escolas: desafios e perspectivas. RGS, 2018;18(2):10-22.

18. SILVA ECA, et al. Conhecimento das mulheres de 18 a 50 anos de idade sobre a importância do exame de Papanicolau na prevenção do câncer de colo uterino no município de Turvânia-GO. Rev. Eletr. Fac. Montes Belos, 2016; 8(4): 101- 202.

19. SISCOLO. Sistema de Informação do câncer do colo do útero. Brasília, 2012. Disponível em: http://w3.datasus.gov.br/siscam/index.php?area=01 Acesso em: 21 jun. 2020.

20. PAULSEN F, WASCHKE J. Sobotta - Atlas de anatomia humana. 24ํe ed. Rio de Janeiro: Guanabara Koogan, 2018.

21. TAVARES PN, et al. A vivência do ser enfermeiro e preceptor em um hospital escola: olhar fenomenológico. Rev Rene, Fortaleza, 2011; 12(4):798-807.

22. TEIXEIRA E. Em tempos de novas Diretrizes Curriculares Nacionais (DCN) para o curso de graduação em enfermagem. Rev. Enferm., UFSM, 2017; 7(2). 